\title{
The Protective Role of Hippophae rhamnoides L. on Rat Brain and Liver Tissues Exposed to Cold Plus Immobilization Stress Model
}

\author{
Nensi CAVAK ${ }^{1}$, Mustafa Koray GUMUSTAS ${ }^{1}$, Sema DEMIRCI CEKIC ${ }^{2}$ \\ ${ }^{1}$ Istanbul University-Cerrahpasa, Cerrahpaşa Faculty of Medicine, Department of Medical Biochemistry, Istanbul, Turkey \\ ${ }^{2}$ Istanbul University-Cerrahpasa, Faculty of Engineering, Department of Chemistry, Istanbul, Turkey \\ The liver tissue part of this study was presented as a poster at the "41st FEBS Congress" conducted in 2016 with the title "The effect of rat liver tissue \\ radical metabolism and the protective role of Hippophae rhamnoides L. on cold and immobilization stress model".
}

Corresponding author: Mustafa Koray GUMUSTAS profgumustas@yahoo.com

\section{ABSTRACT}

AIM: To investigate the protective role of Hippophae rhamnoides L. (Sea buckthorn, SBT) in cold plus immobilization stress-induced oxidative and nitrosative stress in rats.

MATERIAL and METHODS: Thirty-two Wistar albino rats were randomly divided into four groups: control (i.p. physiological saline), SBT (i.p. 200 mg/kg/48h SBT extract), stress (i.p. physiological saline; 6-h cold plus immobilization stress) and SBT+stress (i.p. $200 \mathrm{mg} / \mathrm{kg} / 48 \mathrm{~h} \mathrm{SBT;}$ 6-h cold plus immobilization stress). ${ }^{*}$ In liver and brain tissues 3-nitrotyrosine levels were determined by ELISA while total antioxidant capacity, total thiol, total glutathione, total nitrite+nitrate levels, superoxide dismutase and glutathione peroxidase activities were measured using colorimetric methods.

RESULTS: In the SBT+stress group, the total glutathione levels and glutathione peroxidase activities were significantly higher in both tissues, whereas the total nitrite+nitrate levels and superoxide dismutase activities decreased compared with the stress group. The 3-nitrotyrosine levels as oxidative and nitrosative stress markers were found to be significantly higher in SBT+stress group in both tissues than in the control. No significant differences were found between the stress and SBT+stress groups in the liver.

CONCLUSION: The results show that SBT has antioxidant properties against cold plus immobilization stress-induced oxidative and nitrosative stress and that it can be recommended as a natural antioxidant and nutritional supplement.

KEYWORDS: Brain, Liver, Nitrosative stress, Oxidative stress, Sea buckthorn

ABBREVIATIONS: 3-NT: 3-Nitrotyrosine, CAT: Catalase, CUPRAC: Cupric ion reducing antioxidant capacity, ELISA: Enzymelinked immunosorbent assay, GPx: Glutathione peroxidase, GSH: Reduced glutathione, GSSG: Oxidized glutathione, NO: Nitric oxide, PUFAs: Polyunsaturated fatty acids, ROS: Reactive oxygen species, SBT: Hippophae rhamnoides L. (Sea buckthorn), SOD: Superoxide dismutase, TAC: Total antioxidant capacity

\section{INTRODUCTION}

S tress is defined as the total biological reactions to the physical, mental, emotional, internal or external adverse stimuli that tend to disrupt the homeostasis of organisms (21). It has been shown that nitric oxide (NO) and reactive oxygen species (ROS) production is increased and free radical detoxifying enzyme activities such as glutathione peroxidase (GPx), catalase (CAT) and superoxide dismutase (SOD) are reduced under stress conditions. As brain tissue contains large amounts of polyunsaturated fatty acids (PUFAs), the brain is vulnerable to free radical attacks and these reactions 
lead to structural and functional damage to the cell membrane $(9,15)$.

To investigate stress, different animal models have been developed and frequently used to evaluate the anti-stress activities of natural or synthetic compounds. Various physical stress models are based on changes in body temperature. The combination of different stress factors is considered a better stimulant than a single factor (13). For this reason, in this study, stress was induced by the cold plus immobilization method.

Hippophae rhamnoides L. (Sea buckthorn, SBT) has antioxidant effects against stress-induced oxidative damage throght the nutritional value of its content $(7,19)$. All parts of SBT are considered a source of numerous bioactive substances $(3,8,11,25,31)$. To the best of our knowlage, no preclinical study investigated the protective role of SBT, especially in nitrosative stress in rat brain tissue induced by cold plus immobilization exposure.

Thus, this study aimed to evaluate the efficacy of SBT content in both the liver and brain tissues of rats against oxidative and nitrosative stress induced by an experimental cold plus immobilization stress model. In other words, this study demonstrated the protection of living organisms from oxidative and nitrosative stress, especially in brain tissues, through to supplementation of SBT, which has a high antioxidant content and is a natural herbal product.

\section{MATERIAL and METHODS}

\section{SBT Preparate and Chemicals}

SBT oil extract (Omega-7 SBA24) was supplied commercially by Pharma Nord (Northumberland, UK). It was prepared from both berry pulp and seeds using a supercritical $\mathrm{CO}_{2}$ extraction method. The extracted oil is a rich source of omega-7 fatty acids, including palmitoleic acid $(240 \mathrm{mg} / \mathrm{ml})$ and vaccenic acid $(60 \mathrm{mg} / \mathrm{ml})$. SBT oil also contains oleic acid $(180 \mathrm{mg} / \mathrm{ml})$, linoleic acid (170 mg/ml), a-linolenic acid (130 mg/ml), vitamin $\mathrm{E}$ as a-tocopherol $(4 \mathrm{mg} / \mathrm{ml})$ and vitamin $A$ from $\beta$-carotene $(400 \mu \mathrm{g} / \mathrm{ml})$. Other chemicals were supplied by Sigma-Aldrich (St. Louis, MO, USA).

\section{Animals and Treatments}

All experiments were approved by the ethical committee of Istanbul University (No: 2013/64) and followed the National Institutes of Health Guide for the Animal Care and Use of Laboratory Animals. Animals were obtained from the Experimental Medical Research Institute of Istanbul University.

In this study, male Wistar albino rats, three months of age and weighing $260 \pm 20 \mathrm{~g}$, were used. The rats were housed in a light- and temperature-controlled room on a 12-h light/dark cycle, with standard pellet lab chow and water ad-libitum. After seven days of adaptation, the rats were divided randomly into four groups, with eight rats in each group: control (C), cold plus immobilization stress-exposed (S), SBT-treated (SBT) and SBT-treated + cold plus immobilization stress-exposed $(\mathrm{SBT}+\mathrm{S})$.
In the SBT-treated groups (SBT and SBT+S), the rats were injected with SBT (200 mg/kg/48h) intraperitoneally for 10 days. Instead of SBT, physiological saline $(0.9 \% \mathrm{NaCl})$ was administered intraperitoneally to the rats in control and stress groups. All stress experiments were conducted on healthy rats that fasted overnight. After the last dose of SBT/saline, cold plus immobilization stress procedure was performed between 08:30 and 14:30 on the $11^{\text {th }}$ day in the stress-exposed groups $(S$ and $S B T+S)$. Stress was performed at $+4^{\circ} \mathrm{C}$ by placing each animal in wire cages, which were adjusted to the size of the rat, attached to a slim wooden board, which had a $40 \mathrm{~cm}$ ground clearance (29). At the end of the 6-h stress exposure, all rats were anesthetized with xylazine $(10 \mathrm{mg} / \mathrm{kg})$ and ketamine $(50 \mathrm{mg} / \mathrm{kg})$ intraperitoneally. Brain and liver tissues were immediately isolated and frozen at $-80^{\circ} \mathrm{C}$ until further analysis.

\section{Biochemical Analysis}

Determination of 3-NT and nitrite+nitrate levels in liver and brain tissues

Tissue 3-NT concentrations were assayed the ELISA kit (Hangzou Eastbiopharm, Cat. No: CK-E10766), while nitrite+nitrate levels were determined using the colorimetric assay kit (Roche, Cat. No: 11746081001) in brain and liver tissues.

Determination of total glutathione, total thiol levels and reduced glutathione (GSH): oxidized glutathione (GSSG) ratio in liver and brain tissues

The GSH:GSSG ratio and total glutathione levels were measured using a glutathione assay kit (Cayman, Cat. No: 703002). The total thiol concentrations were assayed by the method of Seldak and Lindsay in tissue homogenates (26).

Determination of antioxidant enzyme (SOD and GPx) activities and total antioxidant capacity (TAC) in liver and brain tissues

The tissue GPx activities were measured using an assay kit (Cayman, Cat. No: 703102), and SOD activities were determined using the method of Sun et al.(27). One unit of SOD was defined as the amount of protein inhibiting the rate of nitro blue tetrazolium reduction by $50 \%$. TAC was measured using the cupric ion reducing antioxidant capacity (CUPRAC) method in brain and liver tissues (22).

\section{Determination of total protein levels in liver and brain tissues}

The concentration of total protein in the samples was determined by the method of Lowry et al. (16).

\section{Statistical Methods}

The data were evaluated using the SPSS 21.0 software, and the results were presented as the mean \pm standard deviation. The experimental groups were compared using the KruskalWallis variance analysis test. Post-hoc analysis was performed by the Mann-Whitney $U$ test, through which significant data were found. $\mathrm{P}<0.05$ was considered statistically significant. 


\section{RESULTS}

Data on the biochemical parameters obtained from the controls and the experimental groups are summerized in Figures 1-3.

\section{Effect of SBT on 3-NT and nitrite+nitrate levels in liver and brain tissues}

The hepatic 3-NT levels were found to increase in the SBT+stress group compared with the controls $(p \leq 0.01)$. No significant differences were found in the other groups $(p>0.05)$ (Figure 1A). A significant increase was observed in the stress and SBT+stress groups compared with the controls in the brain $3-\mathrm{NT}$ levels ( $\leq \leq 0.01, p \leq 0.001$, respectively). The brain 3-NT levels were found to be higher in SBT+stress group than in SBT treatment alone group ( $\leq 0.01)$ (Figure 1B).

The liver total nitrite+nitrate levels were found to be higher in the stress, SBT and SBT+stress groups than in the controls ( $p \leq 0.001, p \leq 0.01, p \leq 0.001$, respectively), whereas decreased nitrite+nitrate concentrations were found in the SBT and SBT+stress groups compared with the stres-exposed group $(p \leq 0.01, p \leq 0.001$, respectively) (Figure $1 C)$. Similarly to liver tissue, a significant increase in the nitrite+nitrate level was found in the stress group compared with the controls in brain tissue $(p \leq 0.001)$. Lower concentraitons were found in the SBT and SBT+stress groups than in the Stress group $(p \leq 0.01$, $p \leq 0.01$, respectively) (Figure 1D).

\section{Effect of SBT on total glutathione, total thiol levels and GSH:GSSG ratio in liver and brain tissues}

The total glutathione levels were decreased in the stress group compared with the controls in liver and brain tissues $(p \leq 0.001$, $\mathrm{p} \leq 0.001$, respectively). Conversely, SBT treatments in the SBT and SBT+stress groups caused a significant elevation of the total glutathione levels in both tissues compared with the stress group $(p \leq 0.001, p \leq 0.001, p \leq 0.001, p \leq 0.01$, respectively) (Figure $2 \mathrm{~A}$ and Figure $2 \mathrm{~B}$ ). A significant decrease was found in the SBT+stress group compared with the SBT group and the control in brain tissue $(p \leq 0.001, p \leq 0.001$, respectively) (Figure 2B).

Similarly, in liver and brain tissues, the GSH:GSSG levels were decreased in the stess group than in the controls $(p \leq 0.001$, $\mathrm{p} \leq 0.001$, respectively). With SBT treatment, elevated levels were detected in the SBT and SBT+stress groups compared with the stress group in both tissues $(p \leq 0.001, p \leq 0.001$, $\mathrm{p} \leq 0.001, p=0.01$, respectively). Moreover, significantly decreased levels were observed in the SBT+stress group compared with the controls and the SBT treatment alone group in the brain tissue $(p \leq 0.001, p \leq 0.001$, respectively) (Figure 2C and Figure 2D).

The hepatic total thiol levels were found to increase more in the stress group than in the controls $(p \leq 0.01)$ (Figure $2 E)$. No significant differences were found in the other groups either tissue ( $p>0.05$ ) (Figure 2E and Figure 2F).

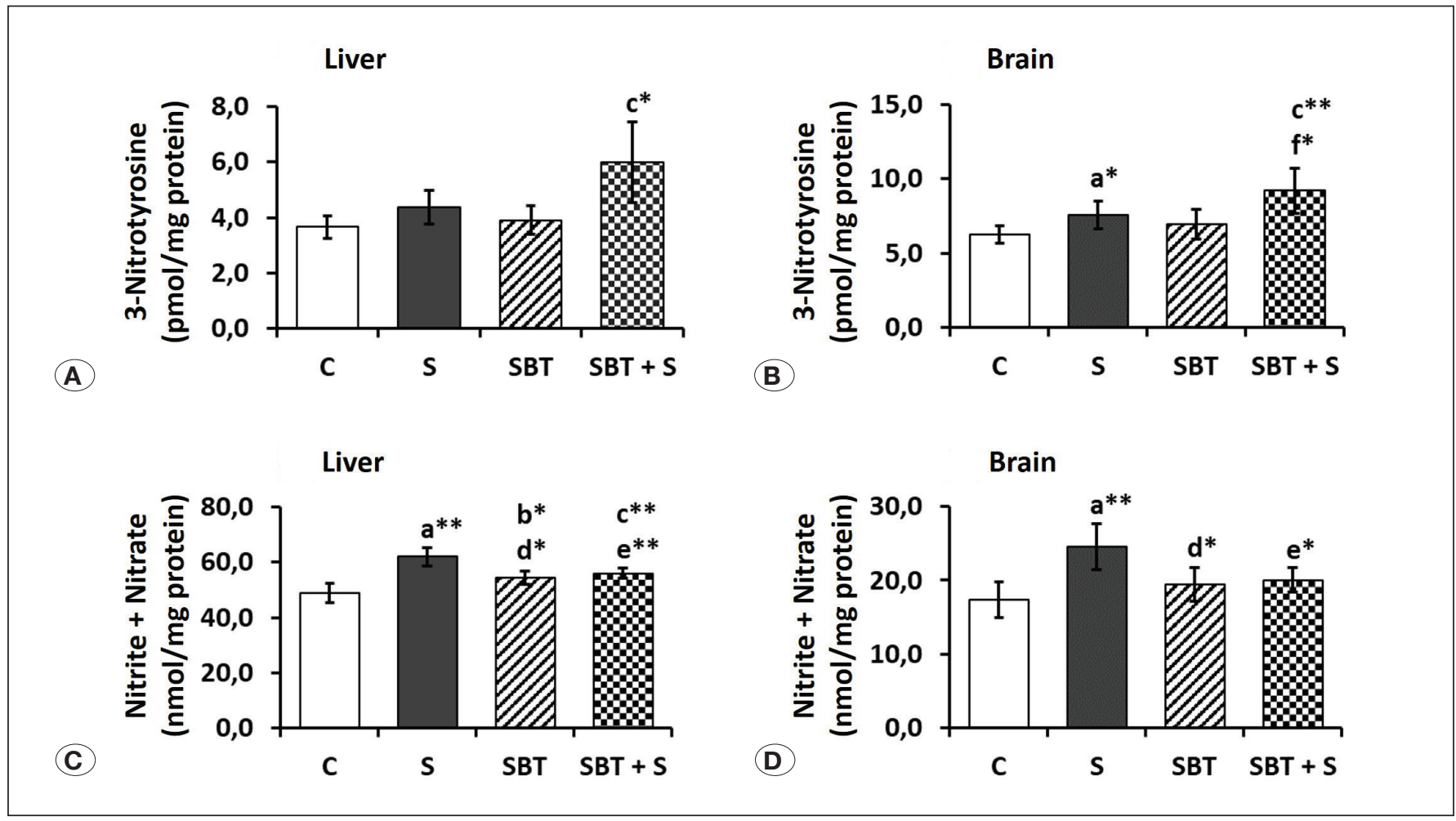

Figure 1: Effects of SBT on nitrosative stress parameters in rat liver and brain tissues. Bar graphs summarizing the 3-NT levels (A, B) and total nitrite+nitrate levels (C, D) in liver and brain tissues. The data expressed as mean \pm SD. Letters (a-f) indicates differences between groups (a,b,c: as compared to the C; $\mathbf{d}$,e: as compared to the S; f: as compared to the SBT). Asterisks $\left(^{*}\right)$ indicates significance $\left({ }^{*} \mathrm{p}<0.01\right.$; $\left.{ }^{* *} p<0.001\right)$. 
Liver

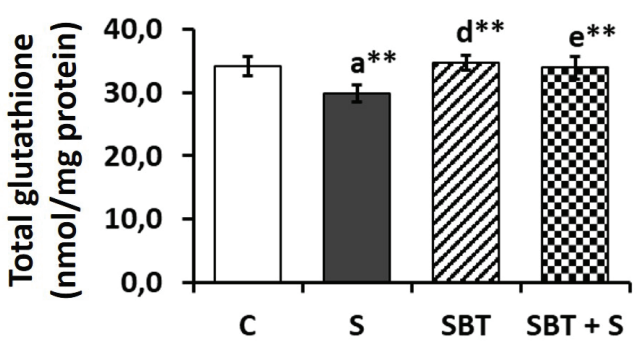

(A)

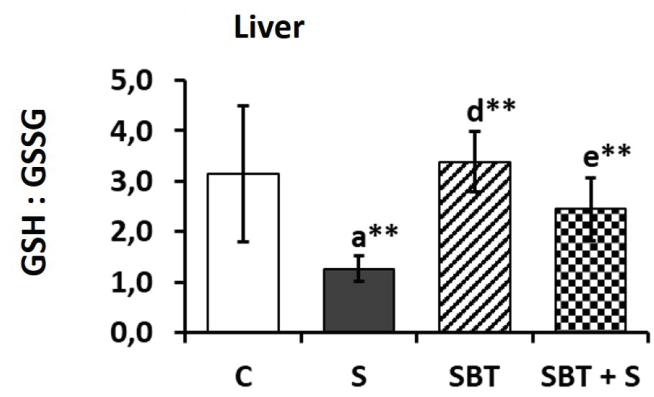

(C)

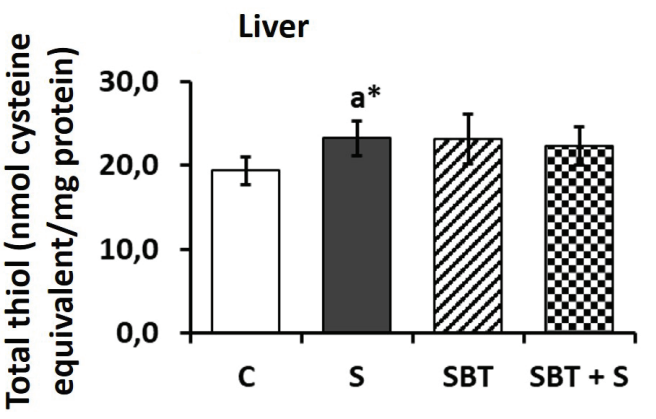

Brain

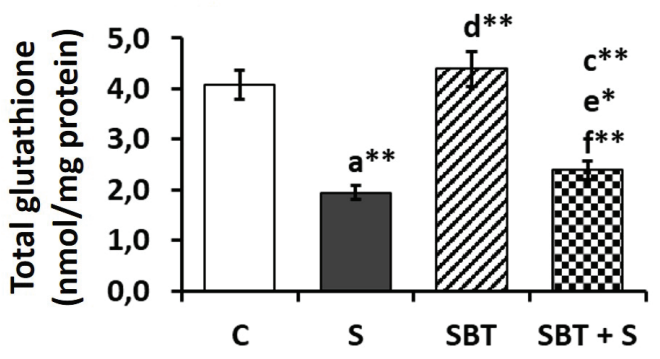

Brain

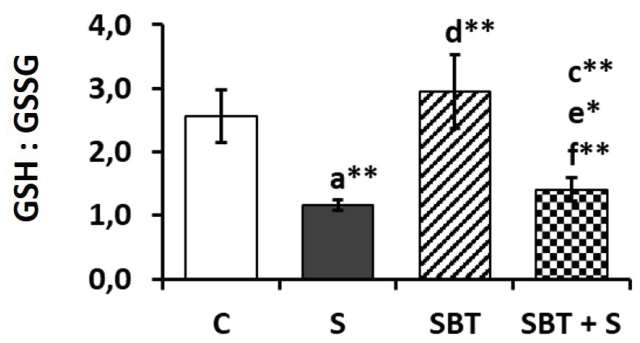

Brain

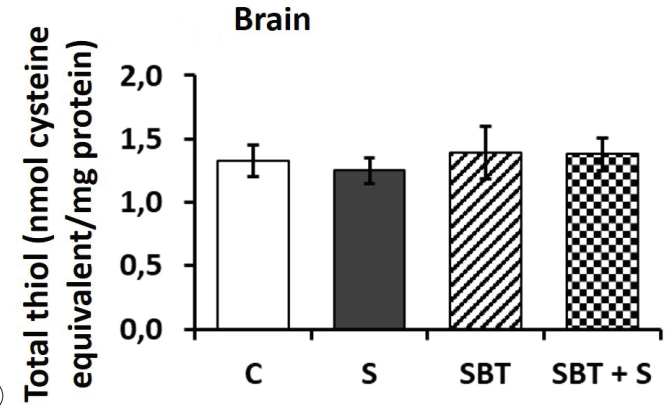

Figure 2: Effects of SBT on glutathione metabolism parameters in rat liver and brain tissues. Bar graphs summarizing the total glutathione levels (A, B), GSH:GSSG ratios (C, D) and total thiol levels (E, F) in liver and brain tissues. The data expressed as mean \pm SD. Letters (a-f) indicates differences between groups (a,b,c: as compared to the C; $\mathbf{d}, \mathbf{e}$ : as compared to the S; f: as compared to the SBT). Asterisks ( $\left.{ }^{*}\right)$ indicates significance $\left({ }^{*} \mathrm{p}<0.01 ;{ }^{* *} \mathrm{p}<0.001\right)$.

Effect of SBT on antioxidant enzyme (SOD and GPx) activities and total antioxidant capacity levels in liver and brain tissues

The hepatic SOD activities were found to increase in the stress group compared with the controls $(p \leq 0.01)$ (Figure $3 A)$. However, a significant decrease was observed in the SBT and SBT+stress groups than in the stress group in the liver and brain tissues ( $\leq \leq 0.001, p \leq 0.01, p \leq 0.01, p \leq 0.01$, respectively) (Figure $3 \mathrm{~A}$ and Figure $3 \mathrm{~B}$ ).

Although the GPx activities were found to be lower in the stress and SBT+stress groups than in the controls in both tissues ( $\leq \leq 0.001, p \leq 0.01, p \leq 0.001, p \leq 0.01$, respectively), the enzyme activities were observed to increase in the SBT and SBT+stress groups compared with the stress group in the liver and brain ( $\mathrm{p} \leq 0.001, \mathrm{p} \leq 0.001, \mathrm{p} \leq 0.001, \mathrm{p} \leq 0.01$, respectively).
Moreover, the GPx activities were lower in the SBT+stress group than in SBT group in both tissues $(p \leq 0.001, p \leq 0.01$, respectively) (Figure 3C and Figure 3D).

In liver tissues, the TAC levels were higher in the stress group than in the controls $(p \leq 0.01)$ and were higher in the SBT group than in the stress group ( $\leq 0.001)$ (Figure $3 E)$. No significant differences were found in brain tissues ( $p>0.05$ ) (Figure 3F).

\section{DISCUSSION}

Different animal models have been developed to induce stress formation, and they are frequently used to evaluate the anti-stress activities of natural and synthetic compounds. In recent years, research on stress has focused on defining new therapeutic approaches and determining the mechanisms of stress response using appropriate animal models $(2,13)$. 
Liver

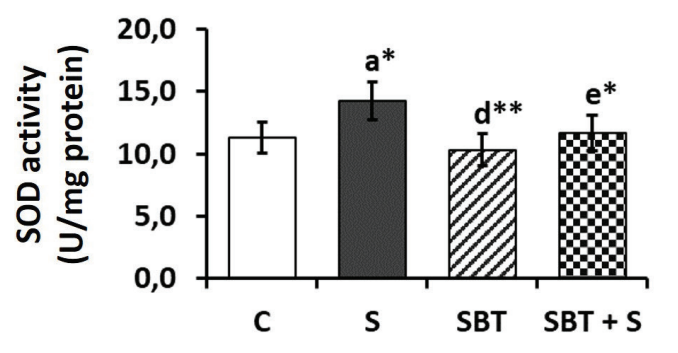

Liver

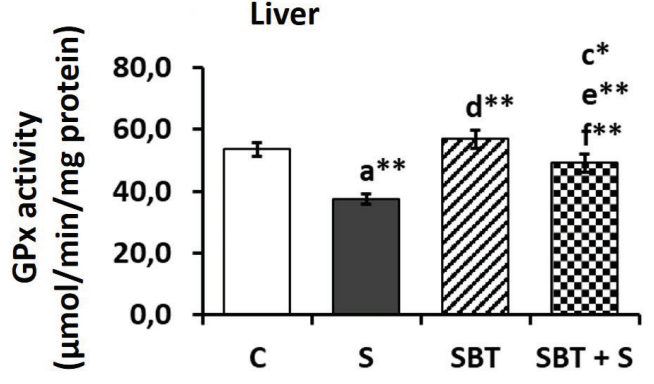

(C)

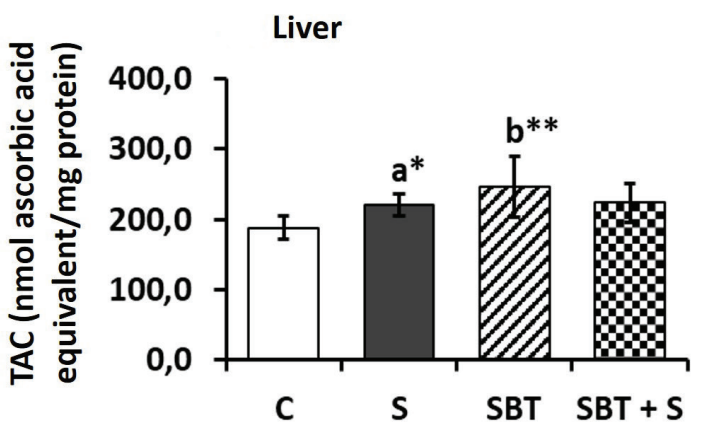

Brain

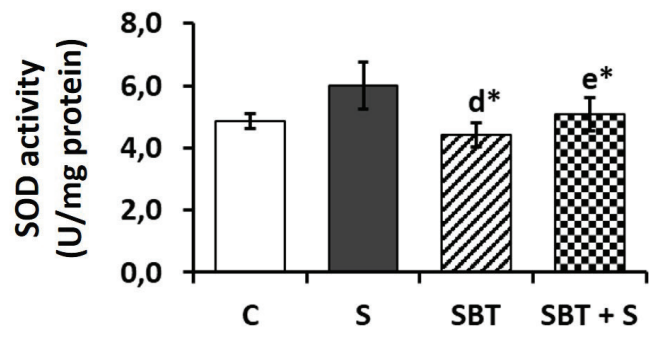

Brain

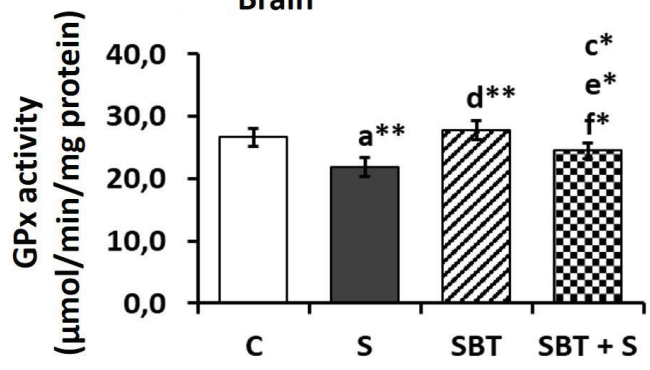

Brain

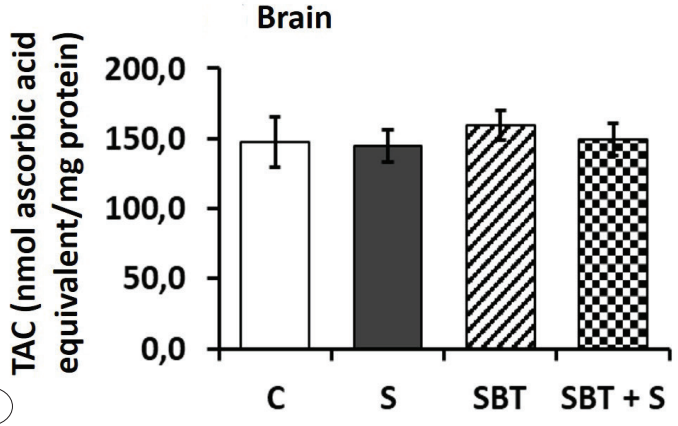

Figure 3: Effects of SBT on antioxidant enzyme activities and total antioxidant capacities in rat liver and brain tissues. Bar graphs summarizing the SOD activities (A, B), GPx activities (C, D) and TAC levels (E, F) in liver and brain tissues. The data expressed as mean \pm SD. Letters $(a-f)$ indicates differences between groups (a,b,c: as compared to the C; $\boldsymbol{d}$,e: as compared to the S; f: as compared to the SBT). Asterisks $\left({ }^{*}\right)$ indicates significance $\left({ }^{*} p<0.01 ;{ }^{* \star} p<0.001\right)$.

Recently, SBT has become an important plant, especially its seed oil, which has high economic value due to its bioactive substances, which can be used in food, medicine, pharmacology and cosmetics. The presence of vitamins, carotenoids, phytosterols, organic acids, PUFAs and some essential amino acids in all parts of SBT enables it to be considered a source of many bioactive substances $(3,14,31)$. SBT is regarded as a medicinal herb that has important value in reducing radiation damage, burns, oral inflammation, and gastric ulcers and in preventing cancer and heart diseases due to its antioxidant phytochemical content and ability to reduce free radical damage. SBT extracts have been documented to improve plasma total cholesterol, triglycerides, LDL and HDL cholesterol levels in hyperlipidemic subjects (10). Various preclinical studies have shown that SBT has antioxidant, immunomodulator, anti-cancer, anti-bacterial, anti-viral, hepatoprotective, anti-stress, adaptogenic, cardioprotective, anti-atherogenic and wound healing activities (28).

In the stress-exposed rat model, endogenous TAC was increased in liver tissue; this could be due to the self-response of organisms against stress. SBT treatment alone induced the highest antioxidant capacity. Because of SBT treatment in stress-exposed group could not increase the antioxidant capacity as much as the only SBT receving group, sufficient exogenous SBT support reduced the need for endogenous antioxidant synthesis.

GSH, a water-soluble antioxidant, is an important lowmolecular-weight tripeptide containing the thiol group in organisms. GSH protects cells against oxidative damage by providing a high redox potential within the cell through its thiol group. GSH is a multifunctional molecule. Aside from being 
a cysteine storage, it is also involved in the detoxification of xenobiotics, the scavenging of free radicals, the regulation of cellular processes such as DNA synthesis and immunological functions, the provision of NO homeostasis, the regulation of post-translational modifications, protein and neurotransmitter receptor activities (17).

When total thiol formation was evaluated in the liver, exposure to stress alone led to total thiol formation as much as in the SBT treatment group. This result shows that the organism can reach an effective total thiol potential against stress.

Total glutathione production was decreased in the stress group compared with the other groups in liver tissue. Therefore, the antioxidant content of SBT may have beneficial effects on the stress response.

The antioxidant-oxidant balance (GSH:GSSG) of liver tissue was found to be significantly lower in the stress-exposed group than in other groups. This result suggests that the most important effect of stress is to enhance the oxidant ratio. However, SBT treatment before stress exposure provided a significant increase in GSH concentration and antioxidantoxidant balance in liver tissue. This shows evidence of the effective antioxidant property of SBT. Moreover, this observation correlates with previous literature that reported that the antioxidant and hepatoprotective activities of the phenolic-rich fraction of SBT leaves in $\mathrm{CCl}_{4}$-induced oxidative stress (19). Our findings are also consistent with those of Saggu and Kumar, who reported that the SBT leaf extract normalized liver GSH levels during cold-hypoxia-restraint stress-induced hypothermia (25).

SOD, a primary antioxidant enzyme against ROS, is a metalloenzyme that catalyzes the dismutation of $\mathrm{O}_{2}{ }^{--}$to $\mathrm{H}_{2} \mathrm{O}_{2}$ and $\mathrm{O}_{2}$. This reaction plays a pivotal role in the defense system against oxidative stress. The level of $\mathrm{O}_{2}^{--}$, a powerful initiator of radical chain reactions, is kept under control through this system in cellular compartments. The organism maintains its protective effect by increasing SOD activity when oxidative stress is increased $(5,18)$. Moreover, GPx catalyzes the reduction of $\mathrm{H}_{2} \mathrm{O}_{2}$ or organic hydroperoxides using GSH. Through this function, GPx prevents the initiation and progression of lipid peroxidation (4).

When the oxygen radical detoxification enzymes were evaluated in the liver, SOD activity was found to be induced because of the possible acute radical increase in the stress group. SBT treatment, with its antioxidant capacity, partially scavenged the radicals and significantly reduced the requirement for SOD. Consistent with SOD, as a secondary antioxidant enzyme, GPx activity was adversely affected because of oxidative damage, and decreased enzyme activities were detected because of stress exposure in liver tissue. Nevertheless, SBT treatment was able to normalize GPx activities approximately. This result suggests that SBT may participate in antioxidant activities by promoting the secondary oxygen radical detoxification mechanism. Consistent with our results, the aqueous fruit extracts of SBT were found to restore SOD, GPx activities, and GSH levels against arsenic-induced toxicity in rat liver tissue (12), and the pre-feeding of an SBT leaf based herbal formulation tended to normalize hexachlorocyclohexane-induced oxidative stress and toxicity and improve the glutathione system (24).

$\mathrm{NO}$ leads to the formation of reactive nitrogen species due to the reaction with $\mathrm{O}_{2}{ }^{--}$or $\mathrm{O}_{2}$. The overproduction of these intermediate products causes toxic results by producing oxidative and nitrosative stress. Nitrosative stress triggers several structural and functional disorders in organism due to nitrosylation reactions and interactions with proteins and other cell structures $(9,30)$. When liver nitrosative stress was evaluated in the stress-exposed group, the significantly increased total nitrite+nitrate levels suggested that the experimental stress model induced nitrosative stress. Nevertheless, SBT treatment provided supportive properties to the antioxidant system by reducing nitrosative stress in liver tissue.

3-NT, a main product and in vivo specific marker of peroxynitrite (ONOO-), is formed through the nitration of the ortho-position of tyrosine amino acid (23). In the liver, the 3-NT levels were found to be significantly higher in the SBT+stress group than in the controls. In the 3-NT correlation with ONOO-, the increased 3-NT levels suggest that SBT seems ineffective and has adverse effects. As $\mathrm{ONOO}^{-}$causes peroxynitrose acid $(\mathrm{ONOOH})$ formation and due to homolytic fusion, toxic products are formed with high reactivity, such as nitrile cation $\left(\mathrm{NO}_{2}{ }^{+}\right)$, nitrogen dioxide radical $\left(\mathrm{NO}_{2}{ }^{\circ}\right)$, and hydroxyl radical $\left(\mathrm{OH}^{\circ}\right)$, leading to the amplification of pro-oxidant activity. According to a previous study investigating the effects of vitamin A in pharmacological doses on the redox state, vitamin A could induce $\mathrm{O}_{2}{ }^{--}$and $\mathrm{ONOO}^{-}$production, and increased 3-NT levels were reported in the rat hypothalamus (6). This finding is consistent with the present study: SBT, with its high antioxidant content including vitamin $A$, could have induced the pro-oxidant activity in the same manner and aggravated nitrosative stress or decreased the NO catabolism rate.

No significant differences were found in the brain total antioxidant capacity and total thiol levels in the stress-exposed group compared with the other groups. This finding suggests that brain tissue may not be affected by stress exposure or may be unqualified to produce antioxidants compared with liver tissue.

Moreover, a decrease was detected in the brain total glutathione levels in the stress group, and the levels per mg of protein were found to be lower than those in liver tissue due to stress exposure. These results suggest that brain tissue is affected more by stress conditions. However, the total glutathione levels, which were significantly increased by the SBT treatment, provided significant antioxidant properties to brain tissue. Experimental animal research and cell culture studies have shown that treatment with the aqueous extract of SBT leaves restored GSH levels, GPx, and SOD activities in hippocampal neurons after hypoxia and that the protective effects were in a dose-dependent manner (20).

Although we did not reach statistical significance, the increase in brain antioxidant SOD activity during stress exposure and the significant decrease in the SBT-treated groups also occured 
in liver tissue. These findings show the protective effect of SBT treatment on both tissues against stress. Moreover, a decrease in the secondary antioxidant enzyme GPx activity in the stress group in brain tissue and a significant increase due to SBT treatments in both the SBT and SBT+stress groups compared with the stress exposed group only indicate that SBT may have supportive effects on the secondary oxygen radical detoxifying mechanism in both brain and liver tissues.

The nitrosative stress in brain tissue was found to increase significantly when the total nitrite+nitrate levels were measured in the stress model. Although the nitrosative stress levels per mg protein were higher in liver tissue, brain 3-NT levels per mg protein were found to be higher than those of liver in the stress group, suggesting that brain tissue could be more affected by oxidative stress. Unlike other tissues, brain tissue has high nNOS activity and NO generation, aside from eNOS and, to a lesser extent, iNOS production (1). This could have contributed to the formation of 3-NT, and its levels correlated with the peroxynitrite concentration that resulted from both nitrosative and oxidative stress, suggesting that increased levels of 3-NT per mg of protein could cause brain damage. Oxidative and nitrosative stress, which were induced by the cold plus immobilization model, significantly increased the total nitrite+nitrate levels in both liver and brain tissues, and the SBT treatments decreased the concentrations. These results indicate that SBT and its antioxidant compounds provide effective protection against stress.

\section{CONCLUSION}

Although SBT causes an increase in liver and brain 3-NT levels, SBT is considered to have supportive effects on the antioxidant system and protective effects against oxidative stress with its high antioxidant content. However, further studies are necessary to evaluate the effects of SBT on oxidative and nitrosative stress and to identify its utilization as a supplement through preclinical studies and clinical trials.

\section{ACKNOWLEDGMENTS}

This work was supported by the Research Fund of Istanbul University-Cerrahpaşa (Project No: 37483).

\section{REFERENCES}

1. Alderton WK, Cooper CE, Knowles RG: Nitric oxide synthases: Structure, function and inhibition. Biochem J 357:593-615, 2001

2. Bali A, Jaggi AS: Preclinical experimental stress studies: Protocols, assessment and comparison. Eur J Pharmacol 746:282-292, 2015

3. Beveridge T, Li TS, Oomah BD, Smith A: Sea buckthorn products: Manufacture and composition. J Agric Food Chem 47:3480-3488, 1999

4. Brigelius-Flohé $\mathrm{R}$, Maiorino $\mathrm{M}$ : Glutathione peroxidases. Biochimica et Biophysica Acta (BBA) - General Subjects 1830:3289-3303, 2013
5. Buettner GR: Superoxide dismutase in redox biology: The roles of superoxide and hydrogen peroxide. Anticancer Agents Med Chem 11:341-346, 2011

6. De Oliveira MR, Oliveira MWS, Da Rocha RF, Moreira JCF: Vitamin A supplementation at pharmacological doses induces nitrosative stress on the hypothalamus of adult Wistar rats. Chem Biol Interact 180:407-413, 2009

7. Geetha S, Singh V, Ram MS, Ilavazhagan G, Banerjee PK, Sawhney RC: Immunomodulatory effects of seabuckthorn (Hippophae rhamnoides L.) against chromium (VI) induced immunosuppression. Mol Cell Biochem 278:101-109, 2005

8. Górnaś P, Šnē E, Siger A, Segliṇa D: Sea buckthorn (Hippophae rhamnoides L.) vegetative parts as an unconventional source of lipophilic antioxidants. Saudi J Biol Sci 23:512-516, 2016

9. Gulati K, Joshi JC, Ray A: Recent advances in stress research: Focus on nitric oxide. Eur J Pharmacol 765:406-414, 2015

10. Guo XF, Yang B, Cai W, Li D: Effect of sea buckthorn (Hippophae rhamnoides L.) on blood lipid profiles: A systematic review and meta-analysis from 11 independent randomized controlled trials. Trends in Food Science \& Technology 61:1-10, 2017

11. Gupta R, Flora S: Therapeutic value of Hippophae rhamnoides L. against subchronic arsenic toxicity in mice. J Med Food 8:353-361, 2005

12. Gupta R, Flora SJ: Protective effects of fruit extracts of Hippophae rhamnoides L. against arsenic toxicity in Swiss albino mice. Hum Exp Toxicol 25:285-295, 2006

13. Jaggi AS, Bhatia N, Kumar N, Singh N, Anand P, Dhawan R: A review on animal models for screening potential anti-stress agents. Neurol Sci 32:993-1005, 2011

14. Jain K, Suryakumar G, Prasad R, Ganju L, Singh SB: Enhanced hypoxic tolerance by Seabuckthorn is due to upregulation of HIF-1a and attenuation of ER stress. J Appl Biomed 14:71-83, 2016

15. Kumar A, Rinwa P, Kaur G, Machawal L: Stress: Neurobiology, consequences and management. J Pharm Bioallied Sci 5:9197,2013

16. Lowry OH, Rosebrough NJ, Farr AL, Randall RJ: Protein measurement with the Folin phenol reagent. J Biol Chem 193:265-275, 1951

17. Lu SC: Regulation of glutathione synthesis. Mol Aspects Med 30:42-59, 2009

18. Lushchak Vl: Free radicals, reactive oxygen species, oxidative stresses and their classifications. Ukr Biochem J 87(6):11-18, 2015

19. Maheshwari DT, Yogendra Kumar MS, Verma SK, Singh VK, Singh SN: Antioxidant and hepatoprotective activities of phenolic rich fraction of Seabuckthorn (Hippophae rhamnoides L.) leaves. Food Chem Toxicol 49:2422-2428, 2011

20. Manickam M, Tulsawani R: Survival response of hippocampal neurons under low oxygen conditions induced by hippophae rhamnoides is associated with JAK/STAT signaling. PLoS One 9:e87694, 2014

21. Monnig AA, Prittie JE: A review of stress-related mucosal disease. J Vet Emerg Crit Care (San Antonio) 21:484-495, 2011 
Cavak N. et al: Hippophea rhamnoides and Stress Model

22. Ozyurek M, Guclu K, Apak R: The main and modified CUPRAC methods of antioxidant measurement. TrAC Trends in Analytical Chemistry 30:652-664, 2011

23. Radi R: Peroxynitrite, a stealthy biological oxidant. J Biol Chem 288:26464-26472, 2013

24. Raghavan AK, Raghavan SK, Khanum F, Shivanna N, Singh BA: Effect of sea buckthorn leaves based herbal formulation on hexachlorocyclohexane-induced oxidative stress in rats. J. Diet Suppl 5:33-46, 2008

25. Saggu S, Kumar R: Modulatory effect of seabuckthorn leaf extract on oxidative stress parameters in rats during exposure to cold, hypoxia and restraint $(\mathrm{C}-\mathrm{H}-\mathrm{R})$ stress and post stress recovery. J Pharm Pharmacol 59:1739-1745, 2007

26. Sedlak J, Lindsay RH: Estimation of total, protein-bound, and nonprotein sulfhydryl groups in tissue with Ellman's reagent. Anal. Biochem 25:192-205, 1968
27. Sun Y, Oberley LW, Li Y: A simple method for clinical assay of superoxide dismutase. Clin Chem 34:497-500, 1988

28. Suryakumar G, Gupta A: Medicinal and therapeutic potential of Sea buckthorn (Hippophae rhamnoides L.). J Ethnopharmacol 138:268-278, 2011

29. Ulakoglu EZ, Gumustas MK, Belce A, Altug T, Kokoglu E: The relationship between endogenous glutathione depletion and energy metabolism in stress-induced gastric mucosal injury. Cerrahpasa J Med 29:127-131, 1998

30. Valko M, Leibfritz D, Moncol J, Cronin MTD, Mazur M, Telser $\mathrm{J}$ : Free radicals and antioxidants in normal physiological functions and human disease. Int J Biochem Cell Biol 39:4484, 2007

31. Yang B, Kallio HP: Fatty acid composition of lipids in sea buckthorn (Hippophaë rhamnoides L.) berries of different origins. J Agric Food Chem 49:1939-1947, 2001 